Communications in Physics, Vol. 26, No. 3 (2016), pp. 209-220

DOI:10.15625/0868-3166/26/3/8956

\title{
THE MODERN INDUCED MATTER APPROACH OF GENERAL RELATIVITY FOR QUANTUM MECHANICS
}

\author{
VO VAN THUAN ${ }^{\dagger}$ \\ Vietnam Atomic Energy Institute (VINATOM), \\ 59 Ly Thuong Kiet street, Hoan Kiem district, Hanoi, Vietnam \\ NGUYEN THI KIM THOA \\ Physics Faculty, Hanoi National University of Education, \\ 136 Xuan Thuy, Dich Vong Hau, Cau Giay, Hanoi, Vietnam \\ ${ }^{\dagger}$ E-mail: vvthuan@vinatom.gov.vn \\ Received 05 September 2016 \\ Accepted for publication 25 January 2017
}

\begin{abstract}
Wesson and his co-workers developed so-called space-time-matter theory (5D-STM) as a generalization of Kaluza-Klein theory, where the extra-dimension in the 5D space-time is no more compacted, but keeping extended in a macroscopic scale to describe the properties of matter in 4D physics. In a trend of 5D-STM approach (or the induced-matter theory), following a bi-cylindrical model of geometrical dynamics, a recent study has shown that the higher $6 D-$ dimensional gravitational equation leads to bi-geodesic description in an extended time-space symmetry which fits Hubble expansion in a "microscopic" cosmological model. As a duality, the geodesic solution is mathematically equivalent to the basic Klein-Gordon-Fock equations of free massive elementary particles. The 4D-embedded dual solutions of the higher dimensional gravitational equation could shed light on origin of physical reality in quantum mechanics, which is to compare with the achievements of the 5D-STM theory.
\end{abstract}

Keywords: time-space symmetry, general relativity, microscopic cosmological model, wave-like solution, Klein-Gordon-Fock equation, physical reality, wave-particle duality.

Classification numbers: 03.65.-w; 04.90.+e; 04.50.Cd.

(C)2016 Vietnam Academy of Science and Technology 


\section{INTRODUCTION}

Following 5D Kaluza-Klein geometrical dynamical theory with extradimension (ED) [1,2], there are two main 5D-approaches with time-like non-compact ED: membrane models in Anti-deSitter geometry (AdS), such as [3,4] and induced matter models [5,6]. In particular, Maldacena [3] found a duality between AdS and conformal fields as AdS/CFT formalism. Randall and Sundrum [4] applied an infinite AdS 5D-model for a hierarchy solution. For the induced matter approach, Wesson [5] has proposed a space-time-matter theory (5D-STM) which developed Kaluza-Klein (K-K) theory with a non-compact ED. The latter should reveal in macroscopic scale not as conventional space-time, but in a specific term, in particular, as the proper mass of an elementary particle. While 10D-supersymmetry and 11D-supergravity models are hardly to have monovalent solutions to contact with 4D-physics, the 5D-STM theory intends not only to unify 4D-gravitation and electromagnetism, but also to serve for interpretation of quantum mechanics. Concerning the membrane models their capability to interpret the aspects of quantum physics remains to be clarified.

Following the induced-matter approach our preliminary study [7] was based on the timespace symmetry in which the Klein-Fock reduction formalism was used $[2,8]$ and the two time-like extra-dimensions were made explicit in terms of the quantum wave function $\psi$ and the proper time variable $t_{0}$. For a next step [9], a duality was found between the quantum wave equations in 4D space-time and a relativistic geodesic description of the curved higher dimensional time-space and, as a result, Heisenberg indeterminism is shown to originate from the space-time curvatures. Concerning the experimental verification, applying 3D-extended time-like curvatures can solve quantitatively the problem of mass hierarchy of charge lepton generations [10] which opens a new possibility for extending to a more general solution of the heavy lepton-neutrino hierarchy. It would be an advantage of our cylindrical dynamical model at variance with more general approaches of other induced matter models. In a recent study [11] by one of the authors the 6Dextended general relativity equation leads to the desirable geodesic equation and its duality with a wave-like solution is able to shed light on physical reality of quantum substances in a more quantitative interpretation than one of the 5D-STM theory. The goal of this study is to introduce those two options of the induced-matter approach and to compare the consequences of the timespace symmetrical model with achievements of the 5D-STM theory in interpretation of quantum mechanics.

The plan of this article is as follows: Except Introduction and Conclusion, there is in Section 2 the Wesson 5D space-time-matter theory is introduced with an emphasis on its application for quantum mechanical interpretation; in Section 3 there is presented bi-cylindrical geometry based on 6D time-space symmetry being applicable for our 6D model; in Section 4 there is shown derivation of dual solutions from 6D time-space gravitational equation; and finally, in Section 5 the quantum mechanical consequences of the 6D time-space model are conducted and compared with ones of the 5D-STM theory.

\section{SPACE-TIME-MATTER THEORY FOR QUANTUM INTERPRETATION}

From the first formulation by Wesson [5] the space-time-matter theory (5D-STM) was proposed later to have a canonical metric form [6] consisting of a separable 4D space-time and an ED 
axis $l$, then its corresponding quadratic 5D-geometry reads:

$$
d S^{2}=g_{A B} d x^{A} d x^{B}=\left[l^{2} / L^{2}\right] d s^{2}+\varepsilon \Phi^{2}\left(x_{\gamma}, l\right) d l^{2},
$$

where $A, B=0 \div 4 ; d s^{2}=g_{\alpha \beta}\left(x_{\gamma}, l\right) d x_{\alpha} d x_{\beta}$ is $4 \mathrm{D}$ space-time sub-geometry; $L$ is a length scale parameter; $\varepsilon= \pm 1$ determining that ED is a space-like $(\varepsilon=-1)$ or time-like $(\varepsilon=+1)$ and $\Phi\left(x_{\gamma}, l\right)$ is a new scalar field, then $g_{44}=\varepsilon \Phi^{2}$. More general, it is possible to replace ED by a shift $l \rightarrow$ $\left(l-l_{0}\right)$ for a constant distance $l_{0}$. As a modern version of $5 \mathrm{D}$ relativity the introduction of the fifth independent dimension has dropped the cylindrical condition of the traditional K-K theory which STM co-workers consider may be daunting algebraically, but this would gain in being richer physically. In 5D-STM theory there was proposed a null geodesic model with $d S^{2}=0$ of extended general relativity in 5D manifolds [6] which is to start from a 5D-Ricci vacuum gravitational equation:

$$
R_{A B}=0,
$$

where $R_{A B}$ is Ricci tensor. Applying Campbell theory [12] to derive the 4D-solutions leads to 4DEinstein gravitational equation and 4D-Maxwell equations of electro-magnetism as it was done in the classical K-K theory. Moreover, new sub-solutions are found additionally in terms of the scalar field $\Phi$. Indeed, from (2) the following solutions are derived:

i) 4D-Gravitational equation:

$$
G_{\alpha \beta}=\frac{k^{2} \Phi^{2}}{2} T_{\alpha \beta}-\frac{1}{\Phi}\left(\nabla_{\alpha} \nabla_{\beta} \Phi-g_{\alpha \beta} \Delta \Phi\right),
$$

where $k=\sqrt{16 \pi G / c^{4}}$ with the gravitational constant $G ; T_{\alpha \beta}$ is energy-momentum tensor; $G_{\alpha \beta}$ is Einstein tensor and $\Delta=g^{\alpha \beta} \nabla_{\alpha} \nabla_{\beta}$ is the wave operator.

ii) Electro-magnetic equations:

$$
\nabla^{\alpha} F_{\alpha \beta}=-3 \frac{\nabla^{\alpha} \Phi}{\Phi} F_{\alpha \beta}
$$

where $F_{\alpha \beta}$ is Faraday tensor.

iii) Scalar field equation:

$$
\Delta \Phi=-\frac{k^{2} \Phi^{3}}{4} F_{\alpha \beta} F^{\alpha \beta} .
$$

The scalar field $\Phi$ would have wide applicability. For example, suggesting $\Phi$ slowly evolving with time, from (3) a cosmological pseudo-constant is determined; from (5) there are some Klein-Gordon-like equations formulated. In the simplest case, when $g_{44}=-\Phi^{2}=-1$, it leads solutions (3) and (4) to 4D-gravitational and Maxwell equations, respectively, exacting ones of the classical K-K theory. Therefore, the 5D-STM theory is a generalization of the traditional 5D $\mathrm{K}-\mathrm{K}$ theory.

The modern 5D K-K theory considered that the cylindrical curvature is a too strong constraint. Instead of this, 5D-STM can get more physical consequences due to non-compactification of ED and more flexible choice of time-space curvature than in the classical K-K theory. In a link with 4D space-time physics of elementary particles the 5D-STM theory would lead to a qualitative interpretation of important issues of quantum mechanics, such as Heisenberg indeterminism $[13,14]$. For example, it was shown that the indeterministic inequality should contain a 
dependence on the fifth dimension, instead of being constrained only by Planck constant:

$$
\left|d p_{\alpha} d x^{\alpha}\right|=n \frac{\hbar}{c}\left(\frac{d l}{l}\right)^{2} .
$$

In $4 \mathrm{D}$ space-time when ED is quantized as $l=n . l_{\min }$ then $\left|d p_{\alpha} d x^{\alpha}\right|=\frac{\hbar}{c} \frac{d n^{2}}{n}$ which resembles Heisenberg inequality. Therefore, the indeterminism in 4D space-time would be governed by a deterministic variation of the fifth dimension in 5D manifold.

There are also a derivation of Klein-Gordon equation from the wave solution of 4D-gravitational equation [15] and the meaning of quantization [16]. An interpretation of waveparticle duality and a wave-like origin of proper mass can be found in [17]. In particular, in [15] Klein-Gordon equation was derived directly from the 5D geodesic equation, which contains an extra-term with constant extra-length $L$. On replacing latter with $\frac{1}{m}$ as the divert mass of particle, this leads to:

$$
\triangle \psi=m^{2} \psi
$$

In fact, there would be a question on the signature of the proper mass $m$ which will be discussed later.

Rich achievements of 5D-STM theory show an encouraging approach to the long-standing problem of consistency between general relativity and quantum mechanics. However, for a decisive solution it needs to search for more quantitative description and interpretation.

\section{TIME-SPACE SYMMETRY BASED CYLINDRICAL GEOMETRY}

At variance with 5D-STM theory, we consider in details the time-space symmetrical model that increases the higher dimensions up to $6 \mathrm{D}$, which would be simplified alternatively by keeping the traditional cylindrical conditions of the EDs. Such a model is preferably applicable for elementary particles, i.e. for quantum substances in scales of microscopic space-time. Namely, in [11] a flat $\{3 T, 3 X\} \equiv\left\{t_{1}, t_{2}, t_{3} \mid x_{1}, x_{2}, x_{3}\right\}$ symmetrical time-space is introduced:

$$
d S^{2}=d t_{k}^{2}-d x_{l}^{2}
$$

where $k, l=1 \div 3$ are summation indices. Here mostly natural units are used throughout except when it needs involving quantum dimension. Similar to the STM theory, the physics of 6Dgeometry is investigated on the 6D-"lightcone" of time-space (8) when $d S=0$, meaning the higher dimensional vacuum:

$$
d t_{k}^{2}=d x_{l}^{2}
$$

In applying the $6 \mathrm{D}$ time-space symmetrical geometry to describe elementary particles, we consider those microscopic time-space fluctuations. The simplest applicable geometry including curved rotation and linear translation is a $\{3 T, 3 X\}$-symmetrical bi-cylindrical geometry. In particular, for an individual fermion elementary particle, e.g. a free lepton with its pseudo-spin $\vec{\tau}$ and/or spin $\vec{s}$ being fixed on an arbitrary axis relative to the longitudinal translational axes of $\left\{t_{k}\right\}$ and $\left\{x_{l}\right\}$, respectively, then the $3 \mathrm{D}$-spherical rotation is reduced to the $2 \mathrm{D}$-cylindrical spinning. This dynamical model leads to a general time-space symmetrical bi-cylindrical geometry:

$$
d \Sigma^{2}=\left(d s_{0}^{2}+d s_{e v}^{2}+d s_{\text {long }}^{2}\right)-\left(d \sigma_{e v}^{2}+d \sigma_{\text {long }}^{2}+d \sigma_{L}^{2}\right)=d t^{2}-d \lambda^{2},
$$

where:

$$
d t^{2}=d \psi\left(t_{0}, t_{k}\right)^{2}+\psi\left(t_{0}, t_{k}\right)^{2} d \varphi\left(t_{0}, t_{k}\right)^{2}+d t_{k}^{2}
$$


and

$$
d \lambda^{2}=d \psi\left(x_{n}, x_{l}\right)^{2}+\psi\left(x_{n}, x_{l}\right)^{2} d \varphi\left(x_{n}, x_{l}\right)^{2}+d x_{l}^{2} .
$$

A contribution from longitudinal fluctuations is expressed through the additional intervals $d s_{\text {long }}$ and $d \sigma_{\text {long }}$ which should be somehow neutralized for conservation of Lorentz invariance. Here the symmetrical curved time-space $\{3 T, 3 X\} \equiv\left\{\psi\left(t_{0}, t_{k}\right), \varphi\left(t_{0}, t_{k}\right), t_{k} \mid \psi\left(x_{n}, x_{l}\right), \varphi\left(x_{n}, x_{l}\right), x_{l}\right\}$. In a semi-phenomenological consideration, the T-odd term $d s_{0}$ is equivalent to a conventional interval of special relativity in $4 \mathrm{D}$-Minkowski space-time, while the P-odd term $d \sigma_{L}$ is a Pnonconserving contribution (PNC) of the weak interaction making a global space-like curvature in term of the left-handed helicity of fermion elementary particles. The P-even term $d \sigma_{e v}$ describes 3D-spatial rotational kinetics around $x_{3}$ being selected as the cylindrical axis and implying that $x_{3} \in\left\{x_{l}\right\}$. The time-like even-term $d s_{e v} \equiv d \sigma_{C P V}$ is introduced to describe CP-violation effects in 3D-time when a past-future variation would be in account. Being embedded in Manifold (9) the cylindrical variables $\psi$ and $\varphi$ are getting functions of linear coordinates $\left\{t_{k}, x_{l}\right\}$ and two 3D-local affine parameters $t_{0}$ and $x_{n}$ which are introduced in according to the longitudinal projection of pseudo-spin $\vec{\tau}$ and spin $\vec{s}$, respectively, namely:

$$
\psi=\psi\left(t_{0}, t_{k}, x_{n}, x_{l}\right)=\psi\left(t_{0}, t_{k}\right) \times \psi\left(x_{n}, x_{l}\right) ; \quad \varphi=\Omega_{0} t_{0}+\Omega_{k} t_{k}-k_{n} x_{n}-k_{l} x_{l}=\Omega_{i} t_{i}-k_{j} x_{j},
$$

where $\{i, j\}$ are summation indices of curved coordinates. In (11) a time-space variable separation is applied for the simplest description of free elementary particles. When (pseudo-)spins $\vec{\tau}$ and/or $\vec{s}$ are fixed along the longitudinal translational axes, the bi-cylindrical geometry is getting simpler without longitudinal components of fluctuation. For an explicit description, $\left\{t_{3}, x_{3}\right\}$ are accepted in Geometry (10) as longitudinal central axes of the bi-cylinder, the curved coordinates of 3D-space are $\left\{x_{j}\right\} \in\left\{x_{1}, x_{2}, z\right\}$ with $k^{2} d z^{2}=k_{n}^{2} d x_{n}^{2}+k_{3}^{2} d x_{3}^{2}$. Similarly, for 3D-time there are $\left\{t_{i}\right\} \in\left\{t_{1}, t_{2}, t\right\}$ where the longitudinal axis $t_{3}$ can combine with the rotational affine parameter $t_{0}$ to form the real physical time $t$ by an orthogonal relationship: $\Omega^{2} d t^{2}=\Omega_{0}^{2} d t_{0}^{2}+\Omega_{3}^{2} d t_{3}^{2}$.

Furthermore, in the following scenario we make an assumption that due to interaction of a Higgs-like potential the time-space symmetry is spontaneously broken, leading to formation of energy-momentum, which turns the spherical curvature into an almost exact intrinsic cylindrical curvature, when an "internal observer" can not see any external 3D-subspaces except the involving him curved evolutional axes $\{t, z\}$. In the result, Geometry (10) turns to a more realistic asymmetrical bi-cylindrical geometry:

$$
d \Sigma^{2}=\left(d s_{0}^{2}+d s_{e v}^{2}\right)-\left(d \sigma_{e v}^{2}+d \sigma_{L}^{2}\right)=d t^{2}-d z^{2},
$$

where

$$
d t^{2}=d \psi\left(t_{0}\right)^{2}+\psi\left(t_{0}\right)^{2} d \varphi\left(t_{0}\right)^{2}+d t_{3}^{2}
$$

and

$$
d z^{2}=d \psi\left(x_{n}\right)^{2}+\psi\left(x_{n}\right)^{2} d \varphi\left(x_{n}\right)^{2}+d x_{3}^{2} .
$$

The asymmetrical curved time-space $\{3 T, 3 X\} \equiv\left\{\psi\left(t_{0}\right), \varphi\left(t_{0}\right), t_{3} \mid \psi\left(x_{n}\right), \varphi\left(x_{n}\right), x_{3}\right\}$. The time-like and space-like intervals in Geometry (12) separate into even and odd constituents, which means the corresponding cylindrical accelerations can flip for and back (as an even-term) or can not flip (as an odd-term) in relation to the cylindrical axis. Odd-terms are determined by internal curvatures, while even-terms relate to external curvatures of cylinders embedded in corresponding flat 3D-subspaces. Naturally, an internal observer involved in a spiral rotation, being not able to distinguish this cylindrical curvature, considers its space-time flat. In principle, a free electron 
reserves its angular momentum (spin) correlating with fixed $d \sigma_{e v}$, but in an external laboratory frame because the proper angular momentum is no more observable unless using an appropriate on-line polarization analysis, then $d \sigma_{e v}$ is getting hidden by a geodesic compensation. In the result, all three linear spatial axes $\left\{x_{l}\right\}$ reveals in transformation from the cylindrical frame to 3Dspace laboratory frame. In 4D-Minkowski space-time the P-odd term is too small as $d \sigma_{L} \ll d \sigma_{e v}$, that makes the weakly curved $\left\{x_{j}\right\} \approx\left\{x_{l}\right\}$ which open as it is observable in a flat 3D-space. The time-like even-term $d s_{e v} \equiv d \sigma_{C P V}$ is introduced to describe CP-violation effects in 3D-time. Therefore, an asymmetry of Geometry (12) means that in 4D-Minkowski subluminal space-time $d \sigma_{C P V} \ll d \sigma_{L} \ll d \sigma_{e v} \ll d s_{0}$, that $d \sigma_{C P V}$ and $d \sigma_{L}$ may be ignored. It makes Geometry (12) turned to $4 \mathrm{D}$ space-time geometry as:

$$
d \Sigma^{2} \approx d s_{0}^{2}-d \sigma_{e v}^{2}=d t^{2}-d \psi\left(x_{n}\right)^{2}+\psi\left(x_{n}\right)^{2} d \varphi\left(x_{n}\right)^{2}+d x_{3}^{2},
$$

where despite $d t^{2}=d \psi\left(t_{0}\right)^{2}+\psi\left(t_{0}\right)^{2} d \varphi\left(t_{0}\right)^{2}+d t_{3}^{2}$, this curved axis should be observed as linear time for $4 \mathrm{D}$ space-time observers. In the result, the $3 \mathrm{D}$-time internal curvature is getting absolute and the subluminal physics is involved in evolution along the physical time $t$, in the meantime, $d \sigma_{e v}$ turns to a pseudo-cylindrical interval in an almost linear 3D-space $\left\{x_{j}\right\}$ with a weakly curved residue along axis $z$. The curvatures are described by EDs $\psi$ and $\varphi$ in (11). When there is not a polarizer for observation in 3D-space (when the 4D-observer gets off from an internal observation back to 3D-space), the spatial spinning is compensated by the space-like pseudo-cylindrical curvature that the 3D-space in (13) is getting absolutely flat, such as:

$$
d \Sigma^{2} \approx d s_{0}^{2}=d t^{2}-\left[d x_{1}^{2}+d x_{2}^{2}\right]-d x_{3}^{2} \equiv d t^{2}-d x_{l}^{2},
$$

where $\left[d x_{1}^{2}+d x_{2}^{2}\right]$ is added to explicit that $x_{3} \in\left\{x_{l}\right\}$ in the original flat 3D-space. The $4 \mathrm{D}$ subluminal geometries (13) and (14) fit the charged lepton sector [10].

\section{A DUAL WAVE-LIKE SOLUTION OF GRAVITATIONAL EQUATION}

Suggesting that in both orthonormal subspaces of 3D-time and 3D-space cylindrical curvature is realized. In principle, a perfect analogical formula with 4D-Einstein equation is applicable to the higher-dimensional general relativity with a modification of gravitational constant (see, for example [18]). Therefore, the gravitational equation in $\{3 T, 3 X\}$-vacuum of Geometry (10) reads:

$$
R_{i}^{m}-\frac{1}{2} \delta_{i}^{m} R=0
$$

where $R_{i}^{m}$ and $R$ are Ricci tensors and the scalar curvature, respectively. Hereafter, for the bicylindrical geometry the signatures $\{---+++\}$ are used following a tradition of general relativity in $4 \mathrm{D}$ space-time.

In according to (11), as $\psi=\psi(y)$ and $\varphi=\varphi(y)$ are not independent variables, we assume that the Hubble law of the cosmological expansion is applicable for the bi-cylindrical model of microscopic space-time: $\frac{\partial \psi}{\partial y}=v_{y}=H_{y} \psi$, where $v_{y}$ is expansion rate being proportional to the "microscopic scale factor" $\psi$, while $H_{y}$ is the "microscopic Hubble constant". The symbolical bi-variable $y \equiv\left\{y_{i}, y_{j}\right\}$ is applied for a shorten description of real curved time-space variables. In a time-space separated form the notations of coordinates $y_{i}$ or $y_{j}$ are time-like or space-like, corresponding to the notations for symmetrical curved time-space: $\{3 T, 3 X\} \equiv\left\{\psi\left(t_{0}, t_{k}\right), \varphi\left(t_{0}, t_{k}\right), t_{k} \mid\right.$ $\left.\psi\left(x_{n}, x_{l}\right), \varphi\left(x_{n}, x_{l}\right), x_{l}\right\}$. Indeed, $y$ are bi-variables, because they serve to present time-space pairs 
of similar functions of corresponding time-like and space-like variables in our model. Therefore, for partial differentiation, it is expressed:

$$
\left[\frac{\partial y}{\partial \psi}\right]=\frac{1}{H_{y} \psi}
$$

Equation (15) with the principle of conservation of linear translation (CLT) and Lorentz-like condition has a symmetrical exponential geodesic solution [11] in according to Geometry (10) as following:

$$
-\frac{\partial^{2} \psi}{\partial t^{2}}+\frac{\partial^{2} \psi}{\partial x_{j}^{2}}=-\left[\left(\frac{\partial \varphi}{\partial t_{0}}\right)^{2}-\left(\frac{\partial \varphi}{\partial x_{n}}\right)^{2}\right] \psi
$$

where as differentials $d t_{3}$ and $d t_{0}$, as well as corresponding covariant derivatives are locally orthogonal to each other, their second derivatives are combined together as: $\frac{\partial^{2} \psi}{\partial t^{2}}=\frac{\partial^{2} \psi}{\partial t_{0}{ }^{2}}+\frac{\partial^{2} \psi}{\partial t_{3}{ }^{2}}$; similarly, due to a local orthogonality, for differentials $d x_{l}$ and $d x_{n}$, the second derivatives in 3D-space are also combined: $\frac{\partial^{2} \psi}{\partial x_{j}^{2}}=\frac{\partial^{2} \psi}{\partial x_{n}^{2}}+\frac{\partial^{2} \psi}{\partial x_{l}^{2}}$.

In principle, variables $\{y\}$ can turn as well as $\{y\} \leftrightarrow\{i y\}$ in a mathematical transformation, then Condition (16) turns to:

$$
\left[\frac{\partial y}{\partial \psi}\right]=\frac{-i}{H_{y} \psi}
$$

In the result, Equation (17) leads to another representation of a wave-like solution with $\psi(y \rightarrow$ $i y) \sim e^{i \varphi}=e^{i\left(\Omega t-k_{j} x_{j}\right)}$ as following:

$$
-\frac{\partial^{2} \psi}{\partial t^{2}}+\frac{\partial^{2} \psi}{\partial x_{j}^{2}}=\left[\left(\frac{\partial \varphi}{\partial t_{0}}\right)^{2}-\left(\frac{\partial \varphi}{\partial x_{n}}\right)^{2}\right] \psi
$$

If time-space symmetry is absolute, the right side is vanished and Equation (19) turns to:

$$
-\frac{\partial^{2} \psi}{\partial t^{2}}+\frac{\partial^{2} \psi}{\partial x_{j}^{2}}=0
$$

Being involved in metrics $g_{\varphi \varphi}$ the functional parameter $\psi$ characterizes time-space curvatures. Then Equation (20) would describe a specific kind of microscopic gravitational waves transmitting with the speed of light. However, the time-space symmetry can never be absolute: we have already assumed that the acceleration term in 3D-time is dominantly enhanced due to interaction with a Higgs-like potential, that will produce a time-space asymmetrical polarization $P \rightarrow P^{+}$.

Qualitatively, the original $\{3 T, 3 X\}$ time-space symmetry is broken spontaneously:

$\left(V_{T} P\right)^{2}=\left[V_{T}\left(\frac{\partial \varphi}{\partial t_{0}^{-}}+\frac{\partial \varphi}{\partial t_{0}^{+}}\right)\right]^{2} \psi \equiv\left[f_{e}\left(\chi+\phi_{0}\right)\right]^{2} \psi \Rightarrow\left(P^{+}\right)^{2}=\left(\frac{\partial \varphi}{\partial t_{0}^{+}}\right)^{2} \psi \equiv\left(f_{e} \phi_{0}\right)^{2} \psi=m_{0}^{2} \psi$,

where $\chi$ is Higgs field and $\phi_{0}$ is Higgs vacuum; $f_{e}$ is Higgs-lepton coupling constant. The arrow means the moment of fixing polarization, equivalent to a spontaneous breaking of symmetry. Since that the elementary particle as a material point has been involved in an almost absolute time-like cylindrical evolution along the real time $t$. Any human observation along the same local geodesic in 3D-time can not distinguish any spiral evolution because the internal curvature of a cylinder 
is zero. This is the reason explaining why the physical time axis of a freely moving elementary particle is linear in 4D-Minkowski space-time.

After spontaneous breaking of time-space symmetry, Equation (17) determines an asymmetrical bi-geodesic equation with exponential solutions in according to Geometry (12):

$$
\frac{\partial^{2} \psi}{\partial t^{2}}-\frac{\partial^{2} \psi}{\partial x_{j}^{2}}=\left[\Lambda_{T}-\left(\frac{\partial \varphi}{\partial x_{n}}\right)_{\text {even }}^{2}-\Lambda_{L}\right] \psi
$$

where $\Lambda_{L} \equiv\left(\frac{\partial \varphi}{\partial x_{n}^{L}}\right)^{2}$ is a small space-like P-odd "cosmological constant" caused by the global weak interaction leading to the left-handed space. Being originated from Einstein gravitational equation (15), Equation (22) describes the microscopic cosmological evolution of time-space curvatures by its de Sitter-like exponential solutions $\psi=\psi_{0} e^{ \pm \varphi}=\psi_{0} e^{ \pm\left(\Omega t+k_{j} x_{j}\right)}$.

Correspondingly, the wave-like equation (19) with $\psi_{w}(y) \equiv \psi(i y)=\psi_{0} e^{ \pm i \varphi}=\psi_{0} e^{\mp i\left(\Omega t-k_{j} x_{j}\right)}$ due to breaking symmetry leads to:

$$
-\frac{\partial^{2} \psi}{\partial t^{2}}+\frac{\partial^{2} \psi}{\partial x_{j}{ }^{2}}=\left[\left(\frac{\partial \varphi}{\partial t_{0}^{+}}\right)^{2}-B_{e}\left(k_{n} \cdot \mu_{e}\right)_{\text {even }}^{2}-\left(\frac{\partial \varphi}{\partial x_{n}^{L}}\right)^{2}\right] \psi .
$$

where $B_{e}$ is a calibration factor and $\mu_{e}$ is magnetic dipole moment of charged lepton; its orientation is in correlation with spin vector $\vec{s}$ and being P-even.

\section{WHAT IS NEW FOR INTERPRETATION OF QUANTUM MECHANICS?}

In mathematical transformation from the exponential solution to the wave-like one, we should change the signature in Equation (22), keeping the wave equation (23) mathematically equivalent to the former. This is realized by transformation of variables: $t \rightarrow-i t$ and $x_{j} \rightarrow i x_{j}$, as well as of their corresponding covariant derivatives: $\frac{\partial f}{\partial t} \rightarrow i \frac{\partial f}{\partial t}$ and $\frac{\partial f}{\partial x_{j}} \rightarrow-i \frac{\partial f}{\partial x_{j}}$, similarly as being adopted for quantum dynamic operators. This procedure is not only a mathematical formalism, but also a significant physical operation, equivalent to transformation from an external observation to an internal investigation. Really, it is the fact in quantum mechanics that the phase velocity of de-Broglie waves in the internal phase continuum is superluminal. Somehow, it is equivalent to converting the role of space $\Leftrightarrow$ time in the internal superluminal frame comparing with the external subluminal space-time. Indeed, instead of the real time in 4D-Minkowski geometry one can use an imaginary time in the corresponding 4D pseudo-Euclid representation. The latter with spacetime $\{x,-i t\}$ is explicitly symmetrical in a mathematical transformation $\{x,-i t\} \Leftrightarrow\{i x, t\}$, but for an observation in the subluminal frame the imaginary coordinate is equivalent to a time axis, while the "real time" can be accepted as a spatial axis. Therefore, by rescaling dynamic action with Planck constant (namely, implementing quantum dynamical operators $\frac{\partial}{\partial t} \rightarrow \hat{E}=i \hbar \frac{\partial}{\partial t}$ and $\frac{\partial}{\partial x_{j}} \rightarrow \hat{p}_{j}=-i \hbar \frac{\partial}{\partial x_{j}}$ ) and making the amplitude of the functional parameter $\psi$ of a scale of Compton length, Klein-Gordon-Fock equation in quantum mechanics is to be formulated explicitly from the wave-like solution (23) of the higher dimensional gravitational equation (15) in the extended time-space of sub-Geometry (12) being approximately closer to sub-Geometry (13) as:

$$
-\hbar^{2} \frac{\partial^{2} \psi}{\partial t^{2}}+\hbar^{2} \frac{\partial^{2} \psi}{\partial x_{j}^{2}}-m^{2} \psi=0
$$


where the square mass term $m$ consists of the following components: $m^{2}=[\hbar \Omega]^{2}=m_{0}^{2}-\delta m^{2}=$ $m_{0}^{2}-m_{s}^{2}-m_{L}^{2}$. Applying Fourier transformation from the phasic space to momentum representation, Equation (24) reads:

$$
E^{2} \psi_{p}-\vec{p}^{2} \psi_{p}-m^{2} \psi_{p}=0
$$

It leads to the relation $E^{2}-\vec{p}^{2}=m^{2}>0$, then Equation (25) describes subluminal motion of an elementary particle with energy $E$ and momentum $\vec{p}$. In comparison with the traditional expression of the rest mass, the present one includes an additional correction $\delta m$ associated with the contribution of the intrinsic spin in 3D-space. The P-even contribution $m_{s}$ linked with an external curvature of spinning in 3D-space can be compensated in according to 3D-spatial local geodesic condition when only the linear translation along $x_{l}$ axis is taken into account for a laboratory frame observation. However, due to P-odd effect being observable in the weak interaction, the geodesic deviation of the material point by its spinning still induces a small non-zero mass factor $m_{L} \ll m_{s}$ which proves a tiny internal curvature of our realistic 3D-space. The latter, in similar to the time-like cylindrical curvature of real time $t$, is not observable from the human point of view of 4D space-time observers, being involved as well in the same global internal cylindrical curvature caused by the weak interaction. In general, Equation (24) is reminiscent of the squared Dirac equation of lepton [7]. In case when there is no polarization analysis, $m \rightarrow m_{0}$, Equation (24) turns to the traditional Klein-Gordon-Fock equation in the linear 3D-space of sub-Geometry (14) (with $\left\{x_{j}\right\} \rightarrow\left\{x_{l}\right\}$ ):

$$
-\hbar^{2} \frac{\partial^{2} \psi}{\partial t^{2}}+\hbar^{2} \frac{\partial^{2} \psi}{\partial x_{l}^{2}}-m_{0}^{2} \psi=0
$$

The consistency between the physical reality of an individual elementary particle and the quantum statistical interpretation is a dilemma causing an unsolved philosophical problem. Our proposed model of bi-cylindrical geometrical dynamics would contribute to understanding some issues of this matter.

First of all, it gives a meaning of the traditional quantum dynamical operators as timespace converting transformation in the phasic continuum, where the phase velocity of a massive particle is always faster than light. The specific kind of gravitational waves carrying the functional variable $\psi$ of metrics $g_{\varphi \varphi}$ along with in the phasic continuum should be superluminal in this context, and it would be a reason why ones can not observe directly the quantum wave function $\psi$ except its squared amplitude. Similar to achievements of the 5D-STM theory we succeeded to derive the quantum mechanical Klein-Gordon-Fock equation. However, there are some significant differences: formulation of Klein-Gordon equation in (7) from 5D-canonical metric (1) was done by hand when one defined a signature of the fifth dimension with parameter $\varepsilon= \pm 1$. In our model, the signature turns naturally by the above-mentioned time-space converting transformation under action of the energy-momentum operators. The proper mass of lepton is also generalized, including all physically realistic phenomena while the mass in 5D-STM theory was determined qualitatively by the length parameter $L$.

Secondly, it would shed light on the wave-particle duality of quantum mechanics. Qualitatively, the wave-like sub-equation (23) leads to description of a quantum substance as a non-local object with quantum wave features in $4 \mathrm{D}$ space-time. On the other side, the exponential solution (22) dually describes the same object, but as a material point, i.e. a localized particle following a 
classical geodesics in an extended time-space. A similar conclusion was achieved by 5D-STM theory which derives the dual solutions of 5D-general relativity as a monotonic exponential geodesic and another wave-like oscillation one, even the duality was obtained by hand-changing signature of the fifth dimension.

Thirdly, from the homogeneous $\{3 T, 3 X\}$ bi-geodesic condition it is possible to derive Heisenberg inequalities from space-time curvatures. Indeed, from the bi-geodesic equation (22) there two 3D-local geodesic conditions in 3D-time and 3D-space are derived as shown in [11] which lead to the following local relationships, respectively:

$$
\begin{gathered}
d E_{0} . d t_{0}^{+}=\psi^{-1} d\left(i . \hbar \frac{\partial \psi}{\partial t_{0}^{+}}\right) d t_{0}^{+}=i . \hbar . d \varphi^{2} . \\
d p_{n} . d x_{n}=\psi^{-1} d\left(-i . \hbar \frac{\partial \psi}{\partial x_{n}}\right) d x_{n}=-i . \hbar . d \varphi^{2} .
\end{gathered}
$$

Based on Equation (27) the time-energy inequality is derived:

$$
|\Delta E| \cdot|\Delta t| \geq\left|\Delta E_{0}\right| \cdot\left|\Delta t_{0}\right|>\left|d E_{0}\right| \cdot\left|d t_{0}^{+}\right|=|i . \hbar| . d \varphi^{2} \geq \Delta \varphi_{\min }^{2} \hbar \geq 0 .
$$

Similarly, applying Equation (28) leads to the space-momentum inequality:

$$
|\Delta p| \cdot|\Delta x| \geq\left|\Delta p_{n}\right| \cdot\left|\Delta x_{n}\right|>\left|d p_{n}\right| \cdot\left|d x_{n}\right|=|i . \hbar| . d \varphi^{2} \geq \Delta \varphi_{\min }^{2} \hbar \geq 0 .
$$

The acceleration terms in 3D-local geodesic conditions would be vanished to turn the inequalities in (29) and (30) equal to zero only when space-time is getting flat. For a non-zero curvature, there is assumed that both inequalities adopt the condition $\Delta \varphi_{\text {min }}=\sigma(\langle\varphi\rangle)=\sqrt{2 \pi}$, where due to a statistical observability of the quantum indeterminism, $\sigma$ is a standard deviation of the mean value $\langle\varphi\rangle=2 \pi$ as a minimum of eigenvalues of the quantized azimuthal angle $(\varphi=2 n \pi)$ in an appropriate stochastic (Poisson or Gaussian) statistical distribution. The statistical feature is possibly caused by collective effects of non-localized interaction in 4D space-time between a detector system consisted of both human action and experimental apparatus with the observable microscopic object, however, being distorted in unpredicted way as an individual elementary particle. In the result, this leads to the traditional Heisenberg inequalities:

$$
\begin{aligned}
& |\Delta E| \cdot|\Delta t| \geq 2 \pi \hbar . \\
& |\Delta p| \cdot|\Delta x| \geq 2 \pi \hbar .
\end{aligned}
$$

Therefore, the quantum indeterminism is found to originate from time-like and space-like curvatures, namely, the time-energy inequality is caused by an intrinsic curvature of 3D-time, while the space-momentum inequality is caused by a P-even contribution of spinning in 3D-space of an individual elementary particle. It is in a qualitative agreement with 5D-STM theory which derived also a Heisenberg inequality (6) of quantum correlation confirming that the 4D indeterminism takes an origin from the variation of the fifth dimension. Instead, the derivation of Heisenberg inequalities (31) and (32) in the time-space symmetrical model is obviously more quantitative and covers both time-energy and space-momentum correlations, while there is not clear how 5D-STM approach can reach the origin of a time-energy inequality. 


\section{CONCLUSION}

During his debates on the quantum indeterminism which Einstein considered philosophically unacceptable, he, instead, endorsed to ascribe physical reality of quantum substance to extradimensions. The achievements of 5D-STM theory as a modern generalization of the inducedmatter K-K theory would be a significant realization of Einstein's dream. Following the matterinduced approach, it was found that a special 6D time-space symmetrical model proposed to solve the problem of microscopic reality is able to reach more quantitative interpretation of quantum mechanics. The mathematical complexity of 6D-geometry has been facilitated significantly by applying the traditional cylindrical conditions without compactification. Both 5D-STM and the timespace symmetrical model belong to the modern Kaluza-Klein geometrical dynamics. Accordingly, a higher dimensional gravitational equation in vacuum has a duality: a monotonic exponential solution and another wave-like representation. In case of the time-space symmetrical model, it is able to derive Klein-Gordon-Fock equation of a massive elementary particle from the wave-like solution, it was found that quantum mechanics is a special technique with its quantum dynamical operators for describing de-Broglie waves as the superluminal microscopic gravitational waves in the microscopic phase continuum carrying energy-momentum in the corresponding subluminal macroscopic space-time. On the other side, the monotonic exponential solution leads to a geodesic description of an elementary particle, in particular a massive lepton, as a material point in the extended time-space. In combination, the dual solutions could shed light on origin of the quantum indeterminism and the wave-particle duality. The present geometrical dynamical interpretation of quantum mechanics is in a good agreement with more general, but more qualitative conclusions of the STM group $[14,17]$. For another quantitative interpretation, as in a homogeneity condition the geodesic equation is equivalent to de Sitter-like exponential solutions then it serves for modeling Hubble expansion in the microscopic time-space, in analogue to the standard model of macroscopic cosmology. In particular, the proposed microscopic cosmological model with an extension of time-like EDs to the 3D time-like configurations should correlate strictly with the number three of lepton generations which has been used to solve the mass hierarchy problem of charged leptons [10]. Consequently, findings from time-space symmetry of microscopic substances would demonstrate a reasonable approach to the consistency between quantum mechanics and general relativity.

\section{REFERENCES}

[1] T. Kaluza, Sitz. Preuss. Akad. Wiss. 33 (1921) 966.

[2] O. Klein, Z. f. Physik 37 (1926) 895.

[3] J.Maldacena, Adv. Theor. Math. Phys. 2 (1998) 231.

[4] L. Randall and R. Sundrum, Phys. Rev. Lett. 83 (1999) 4690.

[5] P. S. Wesson and J. Ponce de Leon, J. Math. Phys. 33 (1992) 3883.

[6] B. Mashhoon, H. Liu and P. S.Wesson, Phys. Lett. B 331 (1994) 305, Erratum: Phys.Lett.B 338 (1994) 519.

[7] Vo Van Thuan, Int. J. Mod. Phys. A 24 (2009) 3545.

[8] V. Fock, Z. f. Physik 39 (1926) 226.

[9] Vo Van Thuan, Comm. Phys. 25 (2015) 247, arXiv: gr-qc/1507.00251 (2015).

[10] Vo Van Thuan, arXiv: 1510.04126 [physics.gen-ph] (2015).

[11] Vo Van Thuan, Comm. Phys. 26 (2016) 181, arXiv: physics.gen-ph/1604.05164v2 (2016).

[12] S. S. Seahra, P. S. Wesson, Class. Quant. Grav. 20 (2003) 1321, arXiv: gr-qc/0302015v4 (2003).

[13] P. S. Wesson, arXiv: 0309134v1 [gr-qc] (2003). 
[14] P. S. Wesson, Int. J. Mod. Phys. D 24 (2015) 1530001-1.

[15] P. S. Wesson, Phys. Lett. B 701 (2011) 379.

[16] P. S. Wesson, Phys. Lett. B 706 (2011) 1.

[17] P. S. Wesson, Phys. Lett. B 722 (2013) 1.

[18] P. S. Wesson, Space-Time-Matter: Modern higher-dimensional cosmology, 2nd Edition, World Scientific (Singapore), 2007. 